\title{
Sequential parameterizing affine projection (SPAP) windowing length for acoustic echo cancellation on speech accents identification
}

\begin{abstract}
Echo cancellation has always in the preprocessing steps before the signals are converted to feature vectors and pattern classification. This is always the correct flow of speech identification. Therefore, in order to get the best cleaned signal, the usage of adaptive echo cancellation removed the echo and also the noise which deteriorates the signals and final results during classification process. The concepts of windowing length may improve the cleaned signals acquired after the noise or echo cancellation process is done. By proposing the preconfigured windowing length through sequential technique, the results is giving improvement from normal length of $200 \mathrm{~ms}$ to $400 \mathrm{~ms}$ whereby the results of Word Error Rate (WER), Equal Error Rate (EER) and accuracies can be viewed with increases around 5-10\% of percentage values compared with echoed signal and reduced the WER and EER too with applying of the sequential parameterization (SPAP) technique.
\end{abstract}

Keyword: Echo cancellation; Sequential windowing length; Accuracy rate; Word error rate; Equal error rate 